\title{
CARACTERIZAÇÃO NUTRICIONAL DE BRANQUILHO (Sebastiania commersoniana (Baillon) Smith \& Downs - Euphorbiaceae), CULTIVADO EM SOLO CONTAMINADO POR PETRÓLEO
}

\author{
Tânia Thibes-Rodrigues*, Celina Wisniewski**, Cleusa Bona***, Renato A. Dedecek****, \\ Gedir de Oliveira Santos***** \\ *Bióloga, Dra . - taniathibes@hotmail.com \\ **Enga. Florestal, Dr ${ }^{\mathrm{a}}$, Depto. de Solos e Engenharia Agrícola, UFPR - cewisni@ufpr.br \\ ***Bióloga, Dra ${ }^{\mathrm{a}}$, Depto. de Botânica, UFPR - cleusabona@ufpr.br \\ ****Eng. Agrônomo, Ph.D., Embrapa Florestas - dedecek@cnpf.embrapa.br \\ *****Biólogo, M.Sc., Depto. de Botânica, UFPR - gedir@ufpr.br
}

Recebido para publicação: 13/12/2005 - Aceito para publicação: 23/08/2006

\begin{abstract}
Resumo
O estudo desenvolvido um ano após o incidente do vazamento de petróleo na Refinaria Presidente Getúlio Vargas (PR) objetivou caracterizar nutricionalmente mudas de branquilho, espécie nativa da região e importante na restauração de áreas degradadas. Mudas foram plantadas em campo em solo contaminado, nãocontaminado, biorremediado e em casa de vegetação, num experimento com quatro tratamentos: solo contaminado, não-contaminado, contaminado adubado e não-contaminado adubado. O experimento de campo foi conduzido por 15 meses no campo e 11 meses na casa de vegetação, após os quais as plantas foram cortadas e amostras de folhas e caules foram analisadas para a quantificação dos macro e micronutrientes. Os resultados da análise das folhas do experimento de campo foram estatisticamente significativos quanto aos teores médios de $\mathrm{K}, \mathrm{Mg}$ e Fe. A contaminação parece ter causado uma redução do teor de $\mathrm{K}$ e um aumento de $\mathrm{Mg}$ e Fe. Para o lenho, foram observadas reduções significativas nos teores de $\mathrm{Ca}, \mathrm{Fe}$ e $\mathrm{Al}$, no tratamento com solo contaminado. No experimento em casa de vegetação, a adição de adubo dificultou a análise dos resultados. As folhas do solo contaminado apresentaram menores teores de $\mathrm{Mg}$ comparativamente a todos os outros tratamentos. Os teores de $\mathrm{Mn}$ e $\mathrm{Zn}$ foram maiores no solo contaminado sem adubação. Nos caules das plantas no solo contaminado, os teores de $\mathrm{N}$ foram maiores no tratamento com solo contaminado adubado. Os teores de $\mathrm{Fe}$ nos caules do tratamento com solo contaminado com e sem adubação foram maiores comparativamente ao solo não-contaminado, e para os outros elementos que apresentaram diferenças estatisticamente significativas ( $\mathrm{Mn}$ e $\mathrm{Al}$ ) foi difícil estabelecer um padrão. Os resultados observados podem também estar relacionados ao efeito da grande variabilidade nas características morfológicas, químicas e físicas dos solos do complexo ambiente de várzea estudado.

Palavras-chave: Sebastiania commersoniana; poluição por petróleo; avaliação nutricional.
\end{abstract}

\section{Abstract}

Nutritional status of Sebastiania commersoniana (Baillon) Smith \& Downs - Euphorbiaceae growing on soil contaminated with petroleum. One year after a large oil spill near President Vargas Refinery, in the state of Parana-BR the effect of soil contamination on the nutritional status of $S$. commersoniana (Euphorbiaceae) seedlings was studied. In a field experiment, seedlings were planted on contaminated, not contaminated and biorreclamated soils. In a greenhouse the experiment consisted of four treatments: contaminated soil, not contaminated soil, contaminated soil + fertilizer and not contaminated soil + fertilizer. Seedlings were grown for 15 months at the field experiment and for 11 months in the greenhouse at the end of which plants were cut off and composite samples of leaves and stems were analyzed for macro and micronutrient content. Results for leaves showed statistical differences on average $\mathrm{K}, \mathrm{Mg}$ and $\mathrm{Fe}$ contents. Contamination semmed to result in a decrease in $\mathrm{K}$ and an increase in $\mathrm{Mg}$ and $\mathrm{Fe}$ content. Significant reductions in $\mathrm{Ca}, \mathrm{Fe}$ and $\mathrm{Al}$ contents were also observed in the stems. In the greenhouse experiment fertilizer addition lead to unclear results. A reduction of $\mathrm{Mg}$ and increase in $\mathrm{Mn}$ and $\mathrm{Zn}$ in the leaves on non contaminated soil was observed. In the stems higher $\mathrm{N}$ contents were found in the contaminated soil with addition of fertilizer, and also higher levels of $\mathrm{Fe}$ in contaminated soil regardless of fertilization. The results could have been strongly influenced by the complexity of soil morphological, chemical and physical characteristics of the alluvial plain.

Keywords: Sebastiania commersoniana; petroleum pollution, nutritional status. 


\section{INTRODUÇÃO}

Acidentes ecológicos envolvendo derramamento de petróleo e seus derivados têm sido freqüentes nas últimas décadas no mundo todo. Os efeitos provocados na fauna e na flora logo após o vazamento podem causar tanto estresses abruptos, agudos, de difícil mensuração, quanto crônicos, também complexos em sua avaliação, podendo se passar muitos anos para que as respostas na natureza sejam detectadas.

O petróleo bruto é uma mistura complexa de alcanos e hidrocarbonetos aromáticos com viscosidade variável, contendo em proporções bem menores oxigênio, nitrogênio e enxofre, que podem se combinar de muitas formas (MANCINI, 2002). Num meio poroso como o solo, sua forma de interação com os minerais de argila, a matéria orgânica, a água e os microorganismos responsáveis pela bioconversão dos compostos originais é bastante complexa. Os fenômenos físico-químicos e biogeoquímicos que atuam no solo podem atenuar o efeito contaminante, retardar sua migração ou até resultar na sua remoção do sistema. A intensidade desses processos pode variar com a heterogeneidade da rocha, a velocidade do fluxo subterrâneo, a espessura da zona não-saturada, o conteúdo de matéria orgânica, a mineralogia das argilas, a estrutura do solo, a porosidade e a permeabilidade (AZAMBUJA et al., 2006). A maneira como esses processos afetam a disponibilidade de nutrientes para as plantas é pouco conhecida.

Diversos trabalhos apontam as plantas como bioindicadoras ou "plantas acumuladoras", sensíveis aos poluentes em geral, podendo filtrar ou acumular substâncias minerais, chegando a sofrer alterações metabólicas e afetando órgãos internos, comprometendo assim seu desenvolvimento (BAKER, 1970; CLARK MACLEOD, 1977; DELAUNE; PATRICK JR.; BURESH, 1979; SMITH; DELAUNE; PATRICK JR., 1981; CHUPAKHINA; MASLENNIKOV, 2004).

$\mathrm{Na}$ área da REPAR, sob o efeito da contaminação por petróleo, Bona; Santos (2003) avaliaram o impacto causado no crescimento e no desenvolvimento de sete espécies arbóreas nativas da região. Estudo com a espécie nativa Campomanesia xanthocarpa, efetuado por Mayer (2004), evidenciou reduções nos teores de clorofila (a, b e total); Oliveira (2004) observou um decréscimo na biomassa de Schinus terebinthifolius e Maranho (2004) registrou alterações significativas na estrutura das folhas e do lenho adulto de Podocarpus lamberti. Souza (1999) analisou a altura média, o incremento periódico e a sobrevivência de treze espécies vegetais nativas da região da REPAR, sob o efeito da contaminação por resíduos oleosos de petróleo em solo biorremediado.

Este trabalho teve como objetivo caracterizar nutricionalmente mudas de Sebastiania commersoniana Baillon cultivada em solo contaminado com petróleo (em campo e estufa), com adição ou não de fertilizante comercial, e em solo submetido ao processo de biorremediação (incorporação de calcário calcítico e microorganismos decompositores de hidrocarbonetos). Trata-se de espécie pioneira nas matas ciliares de diversas formações florestais do sul do Brasil, ocorrendo quase que exclusivamente nas planícies aluviais, onde geralmente se torna a espécie dominante, formando $60 \%$ a $80 \%$ do estrato contínuo das florestas de galeria (SMITH; DOWNS; KLEIN, 1988). Além disso, a espécie é muito indicada para compor reflorestamentos mistos destinados à recomposição de áreas degradadas ao longo das margens de rios e reservatórios, devido à preferência por solos hidromórficos e rusticidade (LORENZI, 1992). Também conhecida por branquilho ou branquinho, sua dispersão é ampla embora descontínua, desde os estados de Minas Gerais e Rio de Janeiro até o Rio Grande do Sul, bem como no Paraguai, Uruguai e Argentina.

\section{MATERIAL E MÉTODOS}

O experimento em campo foi conduzido na área pertencente à Refinaria Presidente Getúlio Vargas (Empresa de Petróleos Brasileiros, PETROBRÁS) - REPAR, no município de Araucária, na região centro-sul do primeiro planalto paranaense $\left(25^{\circ} 35^{\prime} 12^{\prime \prime} \mathrm{S}\right.$ e $\left.49^{\circ} 20^{\prime} 45^{\prime \prime} \mathrm{W}\right)$. Nessa área houve um derramamento de petróleo do tipo Cusiana, em julho de 2000. Esse tipo de petróleo tem como propriedades um volume de $0,025 \%$ de água e sedimentos, densidade $0,9817,0,21 \%$ de enxofre total e viscosidade cinemática $\left(40{ }^{\circ} \mathrm{C}\right)$ de $2,316 \mathrm{~mm}^{2} \cdot \mathrm{s}^{-1}$. Quanto à concentração de hidrocarbonetos, é classificado com um perfil parafínico na faixa de $\mathrm{C}_{10}$ e $\mathrm{C}_{14}$, com $71 \%$ de compostos saturados, $18 \%$ de compostos aromáticos, $10 \%$ de resinas e $1 \%$ de asfaltenos (PETROBRÁS, 2003). O teor de metais pesados e outros elementos no petróleo derramado pode ser visto na tabela 1. 
Tabela 1. Teor de elementos químicos no petróleo derramado na área da REPAR onde foi conduzido o experimento.

Table 1. Heavy metals in the oil spill at the experimental area.

\begin{tabular}{lc}
\hline Elementos & Teor $\mathbf{~} \mathbf{m g ~ k g}^{-\mathbf{1}} \mathbf{)}$ \\
\hline Chumbo & $<10$ \\
Zinco & $<2$ \\
Cobre & $<1$ \\
Níquel & 3 \\
Ferro & 3 \\
Manganês & $<2$ \\
Vanádio & 5 \\
Bário & $<5$ \\
Cálcio & 9 \\
Fósforo & $<15$ \\
Silício & $<100$ \\
Cloro & 103 \\
Fonte: Petrobrás (2003) &
\end{tabular}

O experimento de campo foi montado segundo um delineamento em blocos ao acaso (porque foram plantadas mudas de outras espécies além da avaliada no presente trabalho, que corresponderam aos diferentes tratamentos), em que os blocos corresponderam aos solos contaminado (2C), não-contaminado (2NC) e biorremediado (B1). Em cada parcela foram plantadas aleatoriamente 12 mudas de Sebastiania commersoniana (Baillon) Smith \& Downs - Euphorbiaceae, adquiridas no Horto Municipal de Curitiba $(\mathrm{PR})$, com cinco meses de idade e que corresponderiam às repetições. Dessas 12 mudas, descontando-se a mortalidade, foram selecionadas 6 para análise nutricional.

$\mathrm{O}$ solo das áreas $2 \mathrm{C}$ e $2 \mathrm{NC}$ foi classificado como uma associação de Neossolo Flúvico Ta Eutrófico mesoeutrófico A moderado textura média fase relevo plano raso vegetação floresta subtropical higrófila de várzea e campo subtropical higrófilo de várzea moderadamente a bem drenado + Gleissolo Háplico ta Eutrófico Típico mesoeutrófico A proeminente textura argilosa fase relevo plano vegetação floresta subtropical higrófila de várzea mal drenado (SOUZA et al., 2001).

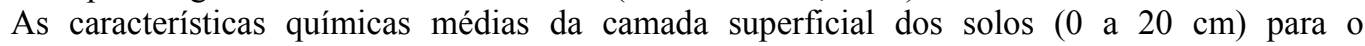
experimento de campo podem ser vistas na tabela 2, cuja determinação seguiu metodologia descrita em EMBRAPA (1997).

Tabela 2. Características químicas do solo nas áreas 2C (contaminada), 2NC (não contaminada) e B1 (biorremediada), na profundidade de 0 a $20 \mathrm{~cm}$.

Table 2. Soil chemical characteristics in the contaminated (2C), not contaminated (2NC) and biorremediated (B1) areas at the $0-20 \mathrm{~cm}$ depth.

\begin{tabular}{|c|c|c|c|c|c|c|c|c|c|c|c|}
\hline \multirow{2}{*}{ Área } & \multirow{2}{*}{$\begin{array}{c}\mathrm{pH} \\
\mathrm{CaCl}_{2}\end{array}$} & Al & $\vec{K}$ & $\mathbf{C a}$ & Mg & CTC & $\mathrm{Fe}$ & Mn & $\mathbf{C u}$ & \multirow{2}{*}{$\begin{array}{c}P \\
\mathrm{mg} \mathrm{dm}^{-3}\end{array}$} & \multirow{2}{*}{$\begin{array}{l}\text { C org. } \\
\mathrm{g} \mathrm{kg}^{-1}\end{array}$} \\
\hline & & \multicolumn{5}{|c|}{ c.mol ${ }_{c} d^{-3}$} & \multicolumn{3}{|c|}{$\mathrm{mg} \mathrm{kg}^{-1}$} & & \\
\hline $2 \mathrm{C}$ & 4,4 & 2 & 0,14 & 2,9 & 2,7 & 19,8 & 703 & 284 & 2,4 & 2,4 & 38,9 \\
\hline $2 \mathrm{NC}$ & 4,1 & 6,5 & 0,19 & 1,5 & 0,9 & 21,6 & 110 & 4 & 1,4 & 2,0 & 38,9 \\
\hline B1 & 6,4 & 0 & 0,22 & 16,8 & 2,2 & 22,9 & 10 & 74 & 0,2 & 1,9 & 29,3 \\
\hline
\end{tabular}

O experimento em casa de vegetação, que também seguiu um delineamento em blocos ao acaso com três tratamentos e 8 repetições (vasos com uma planta), foi realizado no Departamento de Botânica da UFPR. O solo foi trazido do campo das áreas 2C e 2NC para montagem de 4 tratamentos: contaminado (C); não-contaminado (NC); contaminado adubado (CA) e não-contaminado adubado (NCA). O solo foi acondicionado em sacos plásticos de 5 litros, nos quais foram plantadas as mudas de $S$. commersoniana, com 4 meses de idade, adquiridas do CNPF-EMBRAPA. Na tabela 3 podem ser vistas as características químicas do solo com e sem adubação usado na casa de vegetação. A quantidade de adubo utilizada foi calculada a partir dos resultados da análise química, sendo adicionados 0,416 g de cloreto de potássio e 4,16 g de MAP $\left(\mathrm{NH}_{4} \mathrm{H}_{2} \mathrm{PO}_{4}\right.$ na proporção 1:1) para cada $40 \mathrm{~kg}$ de solo. 
Tabela 3. Características químicas do solo do experimento em casa de vegetação, após a adubação para os tratamentos contaminado $(\mathrm{C})$, não contaminado $(\mathrm{NC})$, contaminado adubado $(\mathrm{CA})$ e não contaminado adubado (NCA).

Table 3. Soil chemical characteristics at the greenhouse experiment after fertilization for treatments contaminated (C), not contaminated (NC) and not contaminated with fertilization (NCA).

\begin{tabular}{|c|c|c|c|c|c|c|c|c|c|c|c|c|}
\hline \multirow{2}{*}{ Área } & pH & $\overline{\mathbf{A l}}$ & K & $\overline{\mathbf{C a}}$ & Mg & CTC & \multirow{2}{*}{$\begin{array}{c}P \\
\mathrm{mg} \mathrm{dm}^{-3}\end{array}$} & \multirow{2}{*}{$\begin{array}{l}\text { C org } \\
\mathrm{g} \mathrm{kg}^{-1}\end{array}$} & $\mathrm{Fe}$ & Mn & $\mathbf{C u}$ & $\mathbf{Z n}$ \\
\hline & $\mathrm{CaCl}_{2}$ & \multicolumn{5}{|c|}{ c. $\mathrm{mol}_{\mathrm{c}} \mathrm{dm}^{-3}$} & & & \multicolumn{4}{|c|}{$\mathrm{mg} \mathrm{kg}^{-1}$} \\
\hline $\mathrm{C}$ & 4,5 & 1,2 & 0,28 & 6,6 & 5,6 & 23,2 & 6,5 & 38,3 & 164 & 253 & 3 & 30 \\
\hline $\mathrm{NC}$ & 5,4 & 0 & 0,21 & 7,7 & 6,2 & 19,1 & 4,6 & 27,5 & 115 & 20 & 2 & 15 \\
\hline $\mathrm{CA}$ & 4,4 & 2,9 & 0,32 & 7,2 & 4,8 & 25,4 & 7,0 & 34,1 & 197 & 221 & 3 & 23 \\
\hline $\mathrm{NCA}$ & 4,9 & 0,4 & 0,33 & 8,5 & 5,9 & 22,5 & 10,1 & 22,0 & 212 & 83 & 2 & 5 \\
\hline
\end{tabular}

Nos dois experimentos, as amostras para as análises nutricionais foram compostas de maneira a resultar em 3 repetições tanto para as folhas quanto para o lenho jovem. Esse procedimento foi adotado devido à insuficiência de material disponível para a análise. Foram determinados os teores de macronutrientes ( $\mathrm{N}, \mathrm{P}, \mathrm{K}, \mathrm{Ca}$ e $\mathrm{Mg}$ ), micronutrientes ( $\mathrm{Fe}, \mathrm{Mn}, \mathrm{Zn}$ ) e de alumínio. No experimento de campo a análise foi executada somente nas folhas dos tratamentos $2 \mathrm{C}$ e $2 \mathrm{NC}$, enquanto que para o lenho a análise foi efetuada para os tres tratamentos em função do custo.

As amostras foram secas em estufa a $60^{\circ} \mathrm{C}$ até peso constante, sendo que parte delas foi moída em moinho tipo Wiley e o restante em moinho MR 340 (1984). A digestão das amostras (via seca) foi realizada em mufla (via seca) a $5000{ }^{\circ} \mathrm{C}$, e a solubilização foi feita em ácido clorídrico ( $\left.\mathrm{HCl}\right) 3 \mathrm{~mol} \mathrm{~L}^{-1}$, de acordo com o protocolo de Hildebrand et al. (1976/77). Após filtragem, foram determinados os teores dos macro e micronutrientes das folhas e do caule, seguindo metodologia descrita por Sarruge e Haag (1974). As análises foram feitas no Laboratório de Solos e Nutrição de Plantas do CNPF-EMBRAPA, Colombo (PR), e no Laboratório de Biogeoquímica e Nutrição de Plantas do Departamento de Solos e Engenharia Agrícola da UFPR.

Os dados nutricionais foram analisados com o auxílio do software da Microsoft Excel, seguindo um delineamento em blocos casualizados com três repetições. Os dados foram testados quanto à homogeneidade da variância. Para verificar a significância das diferenças entre os tratamentos, foi efetuada análise da variância. Quando a diferença foi estatisticamente significativa $(\mathrm{p}<0,05)$, foi aplicado o teste de Tukey para comparações múltiplas ao nível de 5\% de probabilidade.

\section{RESULTADOS E DISCUSSÃO}

Os teores médios dos nutrientes analisados nas folhas de $S$. commersoniana podem ser observados na tabela 4. A avaliação de espécies nativas, cuja dinâmica nutricional é pouco conhecida, em geral restringe-se à comparação com a mesma ou outras espécies, se possível crescendo em ambientes semelhantes. Os resultados das análises químicas servem, portanto, muito mais para se caracterizar a espécie estudada do que para se inferir sobre seu estado nutricional, o que exigiria um estudo muito mais aprofundado. Portanto a ênfase da discussão será a comparação entre os teores dos elementos nas plantas do solo contaminado com os teores nas plantas do solo não contaminado. Mesmo assim, devem-se levar em consideração as características específicas da planície aluvial onde o experimento foi conduzido. Em geral, esse tipo de ambiente apresenta uma grande variação espacial na distribuição e nas características morfológicas dos solos em função da proximidade do leito do rio, da variação do nível do lençol freático e do micro-relevo que se forma. Essa variação se confirma com as classes de solo que foram identificadas na área de estudo. Neossolos Flúvicos são formados pela deposição de camadas estratificadas de sedimentos de diferentes granulometrias, e nesse caso não apresentam hidromorfia. Os Gleissolos Háplicos, por outro lado, apresentam horizonte glei começando dentro de $50 \mathrm{~cm}$ da superfície do solo, em função da influência do nível alto do lençol freático. A hidromorfia tem um efeito grande sobre a maioria dos cátions do solo, principalmente na volatilização do nitrogênio e no aumento da disponibilidade do Fe e do $\mathrm{Mn}$, aumentando indiretamente a solubilidade do $\mathrm{P}$ e de outros cátions, como o $\mathrm{K}$, o $\mathrm{Ca}$ e o $\mathrm{Mg}$, que são deslocados para a solução do solo (SOUZA et al., 2006). A grande diferença na concentração de alguns elementos, como por exemplo K, Ca e P no solo contaminado, comparando-se os experimentos de 
campo e de casa de vegetação, confirma a grande variação nas características dos solos que pode ser encontrada nesse tipo de ambiente. Os maiores teores de $\mathrm{P}, \mathrm{Ca}, \mathrm{Mg}, \mathrm{Fe}, \mathrm{Mn}$ e os menores teores de $\mathrm{N}$ no solo contaminado em relação ao solo não contaminado (Tabela 2) poderiam ser um indicativo de condições de maior hidromorfia. O enriquecimento com sedimentos trazidos pelo rio, no caso dos Neossolos Flúvicos, torna ainda mais complexa a dinâmica dos nutrientes nesse tipo de ambiente. Além disso, questões como temperatura de secagem e tempo de armazenamento das amostras têm uma influência grande sobre a extração de micronutrientes (TOMÉ JR., 1997).

Aliado a essa intrincada condição natural, o efeito da presença do óleo em grandes quantidades torna as interações no solo ainda mais complexas. O transporte dos compostos do petróleo se dá pela migração vertical por gravidade e pela dispersão lateral por capilaridade, através da zona não-saturada do solo, sem mistura com o ar ou a água (SANTOS et al., 1998). O petróleo tende a cobrir os grãos com um filme, porque apresenta maior atração pelos sólidos do solo, e, dependendo da quantidade, pode ocupar todos os espaços dos poros (MANCINI, 2002). Esse processo faz parte da chamada atenuação físicoquímica e resulta na formação da fase adsorvida/dissolvida do contaminante que se adere às partículas do solo, principalmente nas argilas de maior atividade. A adsorção/desorção ocorre como conseqüência da reação química entre o íon adsorvido e a superfície sólida adsorvente do poluente. Reações de quelação e complexação também ocorrem, uma vez que o petróleo é um composto orgânico, o que pode facilitar o transporte de metais e a mobilidade de contaminantes orgânicos no subsolo (SANTOS et al., 1998). Portanto, a textura, a mineralogia, o teor de matéria orgânica e o grau de hidromorfia do solo afetam o transporte e a formação da fase adsorvida/dissolvida. Pouco se sabe como os hidrocarbonetos interagem com os cátions adsorvidos aos colóides do solo nesse processo de adsorção/dissolução. Wasserman et al. (2006) concluíram que é possível que a degradação do óleo com o passar do tempo disponibilize sítios de troca para a absorção de metais. A textura dos solos presentes no experimento de campo é bastante diferente. O Neossolo Flúvico apresenta textura média, enquanto a textura do Gleissolo é argilosa, o que sem dúvida deve ter influenciado as interações com o contaminante.

Os teores de $\mathrm{P}, \mathrm{Mn}$ e $\mathrm{Zn}$ apresentaram os maiores coeficientes de variação. Diferenças estatisticamente significativas entre as médias só foram verificadas para os teores de $\mathrm{K}, \mathrm{Mg}$ e $\mathrm{Fe}$.

Os teores de $\mathrm{N}$ para os dois tratamentos e de $\mathrm{P}$ para as mudas do solo contaminado são relativamente superiores aos mencionados por Boeger (2000) para árvores adultas em ambientes tropicais sob as mais diversas condições de solo e altitude, o que torna difícil uma comparação, e também por Souza (1999) na mesma área onde foi realizado este estudo. Deve-se considerar ainda que a espécie em questão foi avaliada no estádio de muda, o que poderia explicar os maiores teores. Wisniewski et al. (1999) avaliando nutricionalmente mudas de Schizollobium parahyba (guapuruvu) e Cithareyilum myrianthum (jacataúva), seis meses após o plantio em ambientes com diferentes níveis de hidromorfia no litoral paranaense, também verificaram teores mais altos de N e P. Também é importante considerar o caráter eutrófico dos solos da área do experimento, ou seja, apresentam uma saturação em bases acima de $50 \%$, o que resultaria numa maior disponibilidade de nutrientes para as plantas. Wassermann et al. (2006) verificaram que a presença do óleo pouco afetou a concentração de $\mathrm{P}, \mathrm{C}$ e $\mathrm{Zn}$ em sedimentos de mangue no Rio de Janeiro.

Houve uma redução no teor de $\mathrm{K}$ nas folhas das plantas crescidas na área contaminada em relação à área não contaminada. Os teores desse elemento no solo contaminado apresentaram-se também menores. Portanto, a diferença pode estar relacionada às características químicas do solo e não à contaminação com o óleo. Os valores de $\mathrm{K}$ registrados encontram-se dentro dos limites (3,5-7,2 g. $\left.\mathrm{kg}^{-1}\right)$ reportados para florestas tropicais úmidas (VITOUSEK; SANFORD, 1986), um pouco abaixo da média, quando comparadas às 12 espécies analisadas na caatinga - 8,95 g. $\mathrm{kg}^{-1}$ (SOBRADO; MEDINA, 1980) - e bem abaixo do teor médio foliar (11,33 g. $\left.\mathrm{kg}^{-1}\right)$ encontrado em Acacia mearnsii por Caldeira et al. (2000).

Os valores de $\mathrm{Mg}$ foram maiores nas folhas do solo contaminado, o que parece estar relacionado com a presença do óleo, uma vez que os teores de $\mathrm{Mg}$ no solo contaminado são muito maiores do que no solo não contaminado (Tabela 2), embora o óleo derramado não apresente alta concentração desse elemento (Tabela 1). Não se pode desconsiderar no entanto o possível efeito da variação da hidromorfia no aumento da concentração desse elemento, que pode ser deslocado para a solução do solo pelo Mn e pelo Fe (SOUZA et al., 2006). 
Tabela 4. Análise nutricional das folhas de S. commersoniana do experimento de campo nas áreas 2C (contaminada) e 2NC (não contaminada).

Table 4. Nutritional contents of the leaves of $S$. commersoniana at the field experiment in the contaminated (2C) and not contaminated (2NC) areas.

\begin{tabular}{lccccccccc}
\hline \multirow{2}{*}{ Área } & $\mathbf{N}$ & $\mathbf{P}$ & $\mathbf{K}$ & $\mathbf{C a}$ & $\mathbf{M g}$ & $\mathbf{F e}$ & $\mathbf{M n}$ & $\mathbf{Z n}$ & $\mathbf{A l}$ \\
\cline { 2 - 9 } & \multicolumn{9}{c}{$\mathbf{g ~ k g}^{-1}$} \\
\hline $2 \mathrm{C}$ & $21,7 \mathrm{a}$ & $0,9 \mathrm{a}$ & $5,2 \mathrm{~b}$ & $9,3 \mathrm{a}$ & $2,5 \mathrm{a}$ & $185 \mathrm{a}$ & $260 \mathrm{a}$ & $25 \mathrm{a}$ & $208 \mathrm{a}$ \\
$2 \mathrm{NC}$ & $21,6 \mathrm{a}$ & $1,7 \mathrm{a}$ & $6,6 \mathrm{a}$ & $10,6 \mathrm{a}$ & $1,9 \mathrm{~b}$ & $77 \mathrm{~b}$ & $177 \mathrm{a}$ & $21 \mathrm{a}$ & $237 \mathrm{a}$ \\
Coef. de variação (\%) & 9,1 & 53,4 & 7,9 & 14,9 & 7,2 & 26,4 & 38,0 & 31,7 & 2,1 \\
\hline
\end{tabular}

Médias seguidas pela mesma letra não diferem estatisticamente entre si pelo Teste de Tukey, ao nível de 5\% de probabilidade.

O maior teor de $\mathrm{Mg}\left(2,42 \mathrm{~g} \cdot \mathrm{kg}^{-1}\right)$ encontrado nas plantas do tratamento contaminado é comparável aos valores (1,4-3,6 $\left.\mathrm{g} \cdot \mathrm{kg}^{-1}\right)$ encontrados em floresta tropical úmida (VITOUSEK; SANFORD, 1986), mas abaixo dos resultados registrados em espécies da restinga paludosa $\left(2,9\right.$ g. $\left.\mathrm{kg}^{-1}\right)$ e muito próximos da média $\left(2,5 \mathrm{mg} \cdot \mathrm{kg}^{-1}\right)$ observada em espécies da restinga seca (BRITEZ, 1994). São, no entanto, bastante inferiores às médias encontradas em três estádios sucessionais de espécies da floresta ombrófila densa das terras baixas (BOEGER, 2000).

$\mathrm{O}$ maior teor de Fe nas folhas sob estresse da contaminação (185 mg.kg $\left.{ }^{-1}\right)$ também está provavelmente relacionado ao seu maior teor no solo contaminado. Esse maior valor não parece estar relacionado à presença do óleo, que contém baixos teores desse elemento, mas provavelmente a um aumento na disponibilização do Fe devido às condições de redução provocadas pelo alagamento do solo. No entanto, Wassermann et al. (2006) consideraram que a presença de óleo em sedimentos de mangue afetou significativamente a disponibilidade biológica do Fe. O mesmo poderia estar acontecendo em relação ao Mn, embora a diferença observada nas folhas não tenha sido estatisticamente significativa.

Os teores de nutrientes nos caules das plantas do experimento de campo estão na tabela 5 . Observa-se que os teores nos caules são menores que os teores foliares (Tabela 4). Teores de nutrientes de caules de plantas jovens dificilmente são reportados na literatura, e por isso a discussão se refere mais à comparação entre os tratamentos. Os teores médios de $\mathrm{Ca}, \mathrm{Mg}, \mathrm{Fe}, \mathrm{Mn}$ e $\mathrm{Al}$ diferiram estatisticamente entre os tratamentos, sem no entanto apresentarem um padrão consistente.

Tabela 5. Análise nutricional dos caules de S. commersoniana em experimento de campo nas áreas 2C (contaminada), 2NC (não-contaminada) e B1 (biorremediada).

Table 5. Nutritional contents of branches of $S$. commersoniana at the field experiment in the contaminated (2C), not contaminated (2NC) and biorremediated (B1) areas.

\begin{tabular}{lccccccccc}
\hline \multirow{2}{*}{ Área } & $\mathbf{N}$ & $\mathbf{P}$ & $\mathbf{K}$ & $\mathbf{C a}$ & $\mathbf{M g}$ & $\mathbf{F e}$ & $\mathbf{M n}$ & $\mathbf{Z n}$ & $\mathbf{A l}$ \\
\cline { 2 - 9 } & \multicolumn{9}{c}{$\mathbf{g ~ k g}^{-1}$} \\
\hline $2 \mathrm{C}$ & $6,5 \mathrm{a}$ & $2,9 \mathrm{a}$ & $5,3 \mathrm{a}$ & $3,7 \mathrm{~b}$ & $0,71 \mathrm{ab}$ & $72 \mathrm{~b}$ & $138 \mathrm{a}$ & $15 \mathrm{a}$ & $92 \mathrm{~b}$ \\
$2 \mathrm{NC}$ & $6,8 \mathrm{a}$ & $5,9 \mathrm{a}$ & $4,5 \mathrm{a}$ & $5,4 \mathrm{a}$ & $0,86 \mathrm{a}$ & $178 \mathrm{a}$ & $141 \mathrm{a}$ & $23 \mathrm{a}$ & $358 \mathrm{a}$ \\
B1 & $6,5 \mathrm{a}$ & $3,7 \mathrm{a}$ & $4,6 \mathrm{a}$ & $5,6 \mathrm{a}$ & $0,58 \mathrm{~b}$ & $111 \mathrm{ab}$ & $24 \mathrm{~b}$ & $15 \mathrm{a}$ & $328 \mathrm{a}$ \\
Coef. de variação (\%) & 9,4 & 33,6 & 12,2 & 12,6 & 13,1 & 30,0 & 14,1 & 18,5 & 26,2 \\
\hline
\end{tabular}

Médias seguidas pela mesma letra não diferem estatisticamente entre si pelo Teste de Tukey, ao nível de 5\% de probabilidade.

$\mathrm{O}$ menor teor de $\mathrm{Ca}$ foi encontrado nos caules de plantas da área contaminada, e os maiores teores médios $\left(5,38-5,64\right.$ g. $\left.\mathrm{kg}^{-1}\right)$ foram encontrados nas plantas das áreas não contaminada e biorremediada, respectivamente. No entanto, ainda se encontram dentro dos valores normalmente reportados para plantas $\left(0,4-15 \mathrm{~g} \mathrm{~kg}^{-1}\right)$ por Larcher (2000).

$\mathrm{O}$ cálcio é absorvido pelas raízes como $\mathrm{Ca}^{+}$, e sua absorção pode ser diminuída por altas concentrações de $\mathrm{K}+$ e de $\mathrm{Mg}^{2}$ no meio (MALAVOLTA, 1980). Essa hipótese pode ser considerada para esse estudo, pois os teores mais elevados de Mg do solo (Tabela 2) e de K nas folhas (Tabela 4) encontram-se na área contaminada e podem ter afetado a absorção de $\mathrm{Ca}$.

$\mathrm{O}$ maior teor de $\mathrm{Ca}$ foi encontrado nos caules das plantas da área biorremediada, que recebeu adição de calcário como parte do processo de remediação, o que resultou num teor mais alto desse 
elemento no solo (Tabela 2). No entanto o teor de $\mathrm{Ca}$ no caule das plantas crescendo no solo não contaminado foi praticamente o mesmo daquele das plantas crescendo na área biorremediada, cujo solo apresentou o menor teor desse elemento.

Os maiores teores de $\mathrm{Mg}$ e Fe foram observados no tratamento não contaminado e também não parecem ter relação com a disponibilidade deles no solo. Os maiores teores de Mn nos tratamentos com e sem contaminação por petróleo também não demonstram essa correlação (Tabela 2). A maior parte do $\mathrm{Fe}$ na planta está localizada nos cloroplastos, e a função do $\mathrm{Mn}$ está envolvida diretamente com a fotossíntese (MARSCHNER, 1995). Portanto, o teor desses elementos nos caules jovens e ainda de coloração esverdeada pode ser bastante variável.

$\mathrm{O}$ menor teor de $\mathrm{Al}$ foi encontrado nas plantas do tratamento contaminado ( $\left.92 \mathrm{mg} . \mathrm{kg}^{-1}\right)$, ao passo que os maiores teores de $\mathrm{Al}$ (328-358 mg. $\left.\mathrm{kg}^{-1}\right)$ encontram-se nos tratamentos biorremediado e nãocontaminado, respectivamente, que foram superiores aos teores encontrados nas folhas. $\mathrm{O}$ teor de $\mathrm{Al}$ nos caules das plantas do tratamento biorremediado, cuja disponibilidade no solo foi nula (Tabela 2), não foi estatisticamente diferente do teor nos caules das plantas do solo contaminado, onde a disponibilidade do elemento foi maior. O alumínio, metal mais abundante da crosta terrestre, está incluído na lista dos metais tóxicos associados a solos com baixo $\mathrm{pH}$, e pouco se conhece sobre sua função fisiológica nos organismos vivos (BEVERIDGE et al., 1997). Existe uma correlação estreita entre a proporção de alumínio trocável no solo, pH e a inibição do crescimento de uma grande variedade de espécies, principalmente de interesse agrícola. Essas interações em solos florestais, principalmente com espécies nativas, são pouco conhecidas. Os teores de $\mathrm{Al}$ no solo não contaminado $\left(6,5 \mathrm{cmol}_{\mathrm{c}} \mathrm{dm}^{-3}\right)$ são bem maiores do que o do solo contaminado $\left(2 \mathrm{cmol}_{\mathrm{c}} \mathrm{dm}^{-3}\right)$, embora o $\mathrm{pH}$ seja apenas um pouco menor. Em ambos os tratamentos, no entanto, o pH é baixo e estaria favorecendo a solubilização do Al, que poderia causar sintomas severos de toxicidade nas plantas (MARSCHNER, 1995). No entanto, o teor nas folhas (Tabela 4) não apresentou diferenças estatisticamente significativas e pode ser considerado baixo. Britez (2001), estudando o efeito do alumínio em duas espécies arbóreas na fase de muda, em solos arenosos da planície litorânea do Paraná, encontrou altas correlações do teor de Al no caule com os parâmetros químicos do solo rizosférico. $\mathrm{O}$ autor considerou o resultado como indicativo de que o aumento dos teores do elemento no solo proporcionou um aumento na absorção e transporte para o caule. $\mathrm{O}$ teor de $\mathrm{Al}$ do caule parece não ter sido afetado pelo aumento no $\mathrm{pH}$ do solo submetido à biorremediação. $\mathrm{O}$ mecanismo de transporte do $\mathrm{Al}$ é pouco conhecido, e os dados obtidos neste trabalho referem-se apenas aos teores e não à quantidade do elemento presente, o que não permite maiores inferências sobre acúmulo ou tolerância da espécie.

Os resultados deste estudo para os teores de Al estão próximos aos valores encontrados por Souza (1999), que trabalhou com solos contaminados com borra de petróleo também na região da REPAR. Em ambos os trabalhos, os menores teores de Al foram encontrados nas plantas sobre solo contaminado por petróleo. Em contrapartida, em experimento conduzido no campo, os maiores teores de alumínio em Campomanesia xanthocarpa (Berg.) foram encontrados quando a espécie se desenvolveu sobre solo contaminado por petróleo (THIBES-RODRIGUES, 2005).

Os resultados das análises químicas das folhas das plantas cultivadas no experimento em casa de vegetação são apresentados na tabela 6 , mostrando que somente os teores médios de $\mathrm{Mg}, \mathrm{Mn}$ e $\mathrm{Zn}$ diferiram estatisticamente entre os tratamentos. Pode-se observar (Tabela 3) que a adubação alterou pouco a disponibilidade do $\mathrm{P}$ e do $\mathrm{K}$ no solo. $\mathrm{O}$ maior aumento de $\mathrm{P}$ ocorreu no solo contaminado comparativamente ao não contaminado. A adubação não afetou o teor desses elementos nas folhas. Os teores de P, são até menores nas folhas de $S$. commersoniana nos tratamentos que receberam adubação. Observou-se também, para todos os elementos, um menor coeficiente de variação comparativamente ao experimento de campo, refletindo uma menor variação das condições de solo e microclima.

$\mathrm{O}$ maior teor de $\mathrm{Mg}$ foi registrado no tratamento com solo não contaminado adubado, embora a fertilização não tenha incluído esse elemento e os teores no solo (Tabela 3) tenham apresentado pequena diferença. $\mathrm{O}$ menor teor de $\mathrm{Mg}$ foi encontrado nas plantas do tratamento contaminado, no entanto, comparativamente aos demais tratamentos a diferença não foi estatisticamente significativa. Os teores podem ser considerados bem inferiores à média considerada ótima $(0,5 \%)$ para o crescimento das plantas por Marschner (1995), em todos os tratamentos. Sintomas visuais de deficiência não foram observados.

O maior teor de Mn (1153 mg. $\mathrm{kg}^{-1}$ ) foi encontrado nas plantas do tratamento contaminado não adubado, e estão dentro da ampla faixa de variação (22-4000 mg. $\mathrm{kg}^{-1}$ ) encontrados em 37 espécies arbóreas analisadas em floresta tropical por Drechsel; Zech (1991), abaixo da média (1968,7 mg. $\mathrm{kg}^{-1}$ )

FLORESTA, Curitiba, PR, v. 36, n. 3, set./dez. 2006 
encontrada para Ilex paraguariensis (erva-mate) estudada por Reissmann et al. (1983) e bastante acima das médias das espécies de três estádios sucessionais da floresta ombrófila densa das terras baixas $(200,21$ a 285,73 mg. $\mathrm{kg}^{-1}$ ) verificadas por Boeger (2000). Comparando os teores foliares com a disponibilidade do elemento no solo, observa-se que parece não haver uma correlação entre os valores. $\mathrm{O}$ solo com menor teor de $\mathrm{Mn}(\mathrm{NC})$ resultou em plantas com maiores teores foliares do elemento. O teor de Mn no solo, por sua vez, pode estar relacionado à variação no $\mathrm{pH}$, uma vez que esse micronutriente, assim como o $\mathrm{Al}$, é solubilizado em solos mais ácidos. Os menores teores no solo correspondem aos tratamentos onde o $\mathrm{pH}$ foi mais alto (NC e NCA). O solo foi retirado do seu ambiente original e submetido a regas artificiais, não se avaliando se as condições redutoras resultantes do alto nível do lençol freático característico de ambiente de várzea permaneceram durante o desenvolvimento do experimento, o que poderia ter afetado a disponibilidade desse elemento. Provavelmente, alguns sítios com ambiente redutor poderiam ter permanecido, considerando a baixa permeabilidade que em geral solos de planície aluvial apresentam, pelo menos os Gleissolos.

Tabela 6. Análise nutricional das folhas de $S$. commersoniana do experimento em casa de vegetação para os tratamentos contaminado $(\mathrm{C})$, não contaminado $(\mathrm{NC})$, contaminado adubado (CA) e não contaminado adubado (NCA).

Table 6. Nutritional contents of the leaves of $S$. commersoniana at the greenhouse experiment for trestments contaminated $(\mathrm{C})$, not contaminated $(\mathrm{NC})$ contaminated with the addition of fertilizer (CA) and not contaminated with the addition of fertilizer.

\begin{tabular}{lccccccccc}
\hline \multirow{2}{*}{ Área } & $\mathbf{N}$ & $\mathbf{P}$ & $\mathbf{K}$ & $\mathbf{C a}$ & $\mathbf{M g}$ & $\mathbf{F e}$ & $\mathbf{M n}$ & $\mathbf{Z n}$ & $\mathbf{A l}$ \\
\cline { 2 - 9 } & & \multicolumn{3}{c}{$\mathbf{g ~ k g}^{-1}$} \\
\hline $\mathrm{C}$ & $22,4 \mathrm{a}$ & $19,7 \mathrm{a}$ & $8,8 \mathrm{a}$ & $15,5 \mathrm{a}$ & $1,7 \mathrm{~b}$ & $523 \mathrm{a}$ & $1126 \mathrm{a}$ & $551 \mathrm{a}$ & $439 \mathrm{a}$ \\
$\mathrm{NC}$ & $22,8 \mathrm{a}$ & $19,0 \mathrm{a}$ & $9,0 \mathrm{a}$ & $16,6 \mathrm{a}$ & $2,4 \mathrm{ab}$ & $538 \mathrm{a}$ & $1153 \mathrm{a}$ & $386 \mathrm{ab}$ & $405 \mathrm{a}$ \\
$\mathrm{CA}$ & $20,3 \mathrm{a}$ & $17,4 \mathrm{a}$ & $9,8 \mathrm{a}$ & $14,8 \mathrm{a}$ & $2,3 \mathrm{ab}$ & $477 \mathrm{a}$ & $890 \mathrm{ab}$ & $336 \mathrm{~b}$ & $295 \mathrm{a}$ \\
NCA & $20,7 \mathrm{a}$ & $16,9 \mathrm{a}$ & $8,7 \mathrm{a}$ & $17,2 \mathrm{a}$ & $2,9 \mathrm{a}$ & $433 \mathrm{a}$ & $785 \mathrm{~b}$ & $398 \mathrm{ab}$ & $367 \mathrm{a}$ \\
Coef. de variação (\%) & 8,2 & 10,6 & 9,1 & 7,3 & 11,7 & 14,0 & 12,9 & 17,7 & 15,4 \\
\hline
\end{tabular}

Médias seguidas pela mesma letra não diferem estatisticamente pelo Teste de Tukey, ao nível de 5\% de probabilidade.

Os resultados das análises dos caules das plantas do experimento em casa de vegetação estão na tabela 7. Os teores médios de N, Fe, Mn, Zn e Al diferem estatisticamente entre os tratamentos. Os teores de todos os elementos, com exceção do $\mathrm{K}$ dos caules do tratamento $\mathrm{CA}$ e do teor de $\mathrm{Al}$ dos tratamentos $\mathrm{C}$ e NCA, foram menores do que os teores foliares.

Tabela 7. Teores de nutrientes e Al nos caules de $S$. commersoniana do experimento em casa de vegetação para os tratamentos contaminado (C), não contaminado (NC), contaminado adubado (CA) e não contaminado adubado (NCA).

Table 7. Nutritional contents of stems of $S$. commersoniana at the greenhouse experiment for the contaminated (C), not contaminated (NC), ) contaminated with the addition of fertilizer (CA) and not contaminated with the addition of fertilizer.

\begin{tabular}{lccccccccc}
\hline \multirow{2}{*}{ Área } & $\mathbf{N}$ & $\mathbf{P}$ & $\mathbf{K}$ & $\mathbf{C a}$ & $\mathbf{M g}$ & $\mathbf{F e}$ & $\mathbf{M n}$ & $\mathbf{Z n}$ & $\mathbf{A l}$ \\
\cline { 2 - 9 } & \multicolumn{3}{c}{$\mathbf{g ~ k g}^{-1}$} \\
$\mathrm{C}$ & $6,9 \mathrm{ab}$ & $13,3 \mathrm{a}$ & $7,8 \mathrm{a}$ & $3,8 \mathrm{a}$ & $0,87 \mathrm{a}$ & $196 \mathrm{ab}$ & $153 \mathrm{a}$ & $95 \mathrm{a}$ & $415 \mathrm{ab}$ \\
$\mathrm{NC}$ & $6,5 \mathrm{ab}$ & $11,4 \mathrm{a}$ & $7,5 \mathrm{a}$ & $3,5 \mathrm{a}$ & $0,89 \mathrm{a}$ & $89 \mathrm{~b}$ & $149 \mathrm{ab}$ & $54 \mathrm{~b}$ & $125 \mathrm{~b}$ \\
$\mathrm{CA}$ & $8,3 \mathrm{a}$ & $13,3 \mathrm{a}$ & $9,3 \mathrm{a}$ & $4,0 \mathrm{a}$ & $0,86 \mathrm{a}$ & $242 \mathrm{a}$ & $160 \mathrm{a}$ & $64 \mathrm{ab}$ & $164 \mathrm{~b}$ \\
$\mathrm{NCA}$ & $5,6 \mathrm{~b}$ & $14,7 \mathrm{a}$ & $6,6 \mathrm{a}$ & $3,0 \mathrm{a}$ & $0,67 \mathrm{a}$ & $201 \mathrm{~b}$ & $87 \mathrm{~b}$ & $49 \mathrm{~b}$ & $531 \mathrm{a}$ \\
Coef. de variação (\%) & 12,5 & 14,1 & 15,0 & 21,3 & 15,4 & 26,6 & 17,1 & 21,7 & 37,9 \\
\hline
\end{tabular}

Médias seguidas pela mesma letra não diferem estatisticamente entre si pelo Teste de Tukey, ao nível de 5\% de probabilidade.

A maior concentração de $\mathrm{Zn}\left(551 \mathrm{mg} \cdot \mathrm{kg}^{-1}\right)$ foi observada nas plantas do tratamento contaminado sem adubo (C). A diferença entre os demais tratamentos não foi estatisticamente significativa. $\mathrm{O}$ solo desse tratamento foi o que apresentou os maiores teores do elemento, embora a variação nos teores dos 
solos dos demais tratamentos seja grande (Tabela 3). Os teores altos de $\mathrm{P}$ no solo poderiam estar causando uma diminuição na absorção do zinco (MALAVOLTA, 1995). No entanto, os teores médios de Zn encontrados nas folhas das plantas jovens de S. commersoniana estão muito acima dos valores (9-101 mg.kg-1) encontrados por Drechsel; Zech (1991) em 37 espécies de floresta tropical, e também acima dos valores mencionados por Larcher (2000), segundo o qual os teores de $\mathrm{Zn}$ nas plantas podem variar entre $1-400 \mathrm{mg} \cdot \mathrm{kg}^{-1}$

Os teores de $\mathrm{N}$ encontrados nos caules jovens das plantas de todos os tratamentos são menores que os valores (12-75 g. $\left.\mathrm{k}^{-1}\right)$ mencionados por Larcher (2000), orém semelhantesao teor médio de $\mathrm{N}(8,00$ g. $\mathrm{kg}^{-1}$ ) encontrado em Acacia mearnsii (com dois anos e quatro meses de idade) pesquisada por Caldeira et aI. (2000). O menor teor de $\mathrm{N}\left(5,59 \mathrm{~g} \cdot \mathrm{kg}^{-1}\right)$ corresponde ao solo não-contaminado adubado. As diferenças entre os demais tratamentos não foram estatisticamente significativas.

$\mathrm{O}$ maior teor de Fe $\left(242 \mathrm{mg} \cdot \mathrm{kg}^{-1}\right)$ foi encontrado nas plantas do tratamento contaminado adubado, e o menor teor ( $\left.89 \mathrm{mg} \cdot \mathrm{kg}^{-1}\right)$ corresponde ao tratamento não-contaminado (Tabela 7). Os maiores teores de $\mathrm{Mn}$ e de $\mathrm{Zn}$ foram encontrados nas plantas dos tratamentos contaminados.

Os teores médios de Al apresentaram uma grande variação entre os tratamentos, e o maior teor foi observado nos caules das plantas do tratamento não contaminado e adubado. Como anteriormente mencionado, teores de nutrientes, principalmente micronutrientes de espécies nativas, em outras partes de plantas que não as folhas, são ainda pouco conhecidos, o que limita a discussão dos resultados observados. Também não ficou clara nenhuma relação dos resultados com os tratamentos, ou seja, os efeitos da contaminação do solo por petróleo sobre o teor de nutrientes nos caules.

Os resultados do experimento do campo mostraram uma maior variação nos teores dos elementos comparativamente à casa de vegetação, em função da impossibilidade do controle das variações ambientais, principalmente no que diz respeito às condições de hidromorfia dos solos, além do estresse da contaminação e da competição pela luminosidade, já que o espaço disponível para o plantio das mudas era restrito, em função do tamanho da área contaminada.

\section{CONCLUSÕES}

No experimento de campo, as folhas das plantas crescendo no solo contaminado apresentaram menores teores de $\mathrm{K}$ e maiores teores de $\mathrm{Mg}$ e Fe. Os caules apresentaram os menores teores de $\mathrm{Ca}, \mathrm{Mg}$, $\mathrm{Fe}, \mathrm{Mn}$ e Al, e os maiores teores de $\mathrm{Mn}$ no solo contaminado em relação ao não contaminado.

No experimento em casa de vegetação, os efeitos da adubação dificultaram a compreensão dos resultados observados em relação à contaminação, tanto para folhas quanto para os caules. As folhas das plantas crescendo no solo contaminado apresentaram menores teores de $\mathrm{Mg}$ comparativamente a todos os outros tratamentos. Os teores de $\mathrm{Mn}$ e $\mathrm{Zn}$ foram maiores no solo contaminado, porém as diferenças entre os demais tratamentos não apresentaram um padrão consistente de variação. Os caules das plantas no solo contaminado com e sem adubação apresentaram maiores teores de Fe comparativamente ao solo não contaminado.

Considerando a complexidade edáfica do ambiente de várzea, os resultados parecem estar mais relacionados à diferença na disponibilidade dos elementos no solo do que ao vazamento do petróleo, embora pouco se conheça sobre os efeitos do óleo nas características químicas do solo.

\section{REFERÊNCIAS}

AZAMBUJA, E.; CANCELIER, D. B.; NANNI, A. Contaminação de solos por LNAPL: discussão sobre diagnóstico e remediação. Porto Alegre. PUCRS. Disponível em: $<$ http://azambuja.com.br/acervo/geosul2000.pdf> Acesso em: 08 jun 2006.

BAKER, J. M. The effects of oils on plants. Environmental Pollution. England: Elsevier. v.1, p.27-44, 1970.

BEVERIDGE, T. J. et al. Metal microbe interactions: contemporary approaches. Advances in microbioal physiology, London, v.38, p.177-243, 1997.

BOEGER, M. R. T. Morfologia foliar e aspectos nutricionais de espécies arbóreas em três estádios sucessionais de floresta ombrófila densa das terras baixas, Paranaguá, PR. 150f. Tese (Doutorado em Ciências Florestais) - Setor de Ciências Agrárias, Universidade Federal do Paraná, Curitiba, 2000. 
BONA, C.; SANTOS, O. G. Adaptações fisiológicas de espécies vegetais crescendo em solo contaminado com petróleo. Curitiba: FUNPAR - UFPR, 2003. Relatório Técnico.

BRITEZ, R. M. Ciclagem de nutrientes minerais em duas florestas da planície litorânea da Ilha do Mel, Paranaguá. 102f. Dissertação (Mestrado em Ciências do Solo) - Setor de Ciências Agrárias, Universidade Federal do Paraná, Curitiba, 1994.

BRITEZ, R. M. Efeito do alumínio em duas espécies arbóreas da planície litorânea da Ilha do Mel, PR. 134f. Tese (Doutorado em Ciências Florestais) - Setor de Ciências Agrárias, Universidade Federal do Paraná, Curitiba, 2001.

CALDEIRA, M. V. W.; SCHUMACHER, M. V.; SANTOS, E. M. dos; TEDESCO, N.; PEREIRA, J. C. Estimativa do conteúdo de nutrientes em um povoamento jovem de Acacia mearnsii De Wild. Estabelecido na região sul do Brasil. Floresta, Curitiba, v.29, n.1/2, p.53-65, 2000.

CHUPAKHINA, G. N.; MASLENNIKOV, P. V. Plant adaptation to oil stress. Russian Journal of Ecology, Moscow, v.35, n.5, p.290-295, 2004. Translated from Ekologiya.

CLARK, R. C.; MAcLEOD, W. O. Inputs, transport mechanisms, and observed concentrations of petroleum in the marine environment. In: MALINS, O. C. (Ed.). Effects of petroleum on arctic and subarctic marine environments and organisms. NewYork: Academic, 1977, v.1, p.91-224.

DELAUNE, R. O.; PATRICK JR., W. H.; BURESH, R. J. Effect of crude oil on Louisiana Spartina alterniflora salt marsh. Environmental Pollution, London, p.21-31, 1979.

DRECHSEL, P.; ZECH. W. Foliar nutrient levels of broad-leaved tropical trees: a tabular review. Plant and Soil, Hague, v.131, p.29-46, 1991.

EMBRAPA. Serviço Nacional de Levantamento e Conservação de Solos. Manual de métodos de análise de solo: v. 1, Rio de Janeiro, 1997. 210p.

HILDEBRAND, C. et al. Manual de análise química de solo e plantas. Curitiba: UFPR, 1976-1977. 255p. Mimeografado.

LARCHER, W. Ecofisiologia vegetal. São Carlos: RiMa, 2000. 531p.

LORENZI, H. Árvores brasileiras: manual de identificação e cultivo de plantas arbóreas nativas do Brasil. Nova Odessa: Plantarum, 1992. 352p.

MALAVOL TA, E. Elementos de nutrição mineral de plantas. São Paulo: Agronômica Ceres, 1980. $251 \mathrm{p}$.

MANCINI, T. M. Métodos de caracterização de áreas potencialmente contaminadas por hidrocarbonetos de petróleo. 187f. Monografia de Graduação (Geologia) - Instituto de Geociências e Ciências Exatas, Universidade Estadual Paulista, Rio Claro, 2002.

MARANHO, L. T. O efeito da poluição por petróleo na estrutura da folha e do lenho de Podocarpus lamberli Klotzsch ex Endl. (Podocarpaceae). 200f. Tese (Doutorado em Ciências Florestais) - Setor de Ciências Agrárias, Universidade Federal do Paraná, Curitiba, 2004.

MARSCHNER, H. Mineral nutrition of higher plants. 2.ed. San Diego: Academic, 1995. 889p.

MAYER, J. L. S. Impacto do solo contaminado com petróleo no crescimento e desenvolvimento de Campomanesia xanthocarpa O. Berg (Myrtaceae). 81f. Monografia (Bacharelado em Ciências Biológicas) - Setor de Ciências Biológicas, Universidade Federal do Paraná, Curitiba, 2004.

OLIVEIRA, L. S. Influência do solo contaminado com petróleo na morfologia e fisiologia de Schinus terebinthifolius Raddi (Anacardiaceae). 63f. Dissertação (Mestrado em Botânica) - Setor de Ciências Biológicas, Universidade Federal do Paraná, Curitiba, 2004.

PETROBRÁS. Caracterização física e química preliminar do óleo derramado na REPAR em julho de 2000. Curitiba, 2003. 2p. Relatório Técnico. 
REISSMANN, C. B. et al. Bioelementos em folhas e hastes de erva-mate (Ilex paraguariensis St. Hill.) sobre cambissolos na região de Mandirituba - PR. Floresta, Curitiba, v.14, n.2, p.49-54, 1983.

SANTOS, P. R. P.; OLIVEIRA NETO, F. A. de; GOMES, Z. L. G. C. Considerações sobre tecnologias para remediação de solos e águas subterrâneas contaminadas e suas aplicações em pólos industriais na região metropolitana de Salvador e na antiga fábrica COBRAC em Santo Amaro - BA. 62p. Monografia (Curso de Especialização em Gerenciamento e Tecnologias Ambientais na Indústria) Departamento de Hidráulica e Saneamento, Escola Politécnica, Universidade Federal da Bahia, Salvador, 1998.

SARRUGE, J.R.; HAAG, H. P. Análises químicas em plantas. Piracicaba: ESALQ/USP, 1974. 56p.

SMITH, C. J.; DELAUNE, R. D.; PATRICK JR., W. H. A method for determining stress in wetland plant communities following an oil spill. Environmental Pollution (Series A), London, n.26, p.297-304, 1981.

SMITH, L. B.; DOWNS, J.; KLEIN, R. M. Euforbiáceas. In: REITZ, P. R. (Ed.). Flora Ilustrada Catarinense. Itajaí: Herbário Barbosa Rodrigues, 1988.

SOBRADO, M. A.; MEDINA, E. General morphology, anatomical structure, and nutrient content of sclerophylous leaves of the "Bana" vegetation of Amazonas, Oecologia, Berlim, v.45, n.3, p.341-345, 1980.

SOUZA, L. C. P. et al. Diagnóstico e monitoramento da cobertura vegetal da área de influência direta e indireta do vazamento de óleo da refinaria Presidente Getúlio Vargas - REPAR, Araucária - PR: fase 1 - diagnóstico. Curitiba: FUNPAR, 2001. Relatório técnico.

SOUZA, S. L. de. Revegetação de área de biorremediação contaminada por resíduos oleosos de petróleo. Curitiba, 1999. 103f. Dissertação (Mestrado em Ciências Florestais) - Setor de Ciências Agrárias, Universidade Federal do Paraná, Curitiba, 1999.

SOUZA, R.; CAMARGO, O. A.; VAHL, L.C. Solos alagados (reações de redox). In: MEURER, E. J. (Ed.). Fundamentos de química do solo. 3.ed. Porto Alegre: Evangraf, 2006. p.185-206.

THIBES-RODRIGUES, T. Os efeitos do solo contaminado com petróleo na estrutura anatômica e estado nutricional do lenho jovem de Campomanesia xanthocarpa Berg (Myrtaceae) e Sebastiania commersoniana (Baillon) Smith \& Downs (Euphorbiaceae). 154f. Tese (Doutorado em Ciências Florestais) - Setor de Ciências Agrárias, Universidade Federal do Paraná, Curitiba, 2005.

THOMÉ Jr., J. B. Manual para interpretação de análise de solo. Guaíba: Agropecuária, 1997. 247p.

VITOUSEK, P. M.; SANFORD Jr, R. L. Nutrient cycling in moist tropical forest. Annual Review of Ecology and Systematics, Palo Alto, v.17, p.137-167, 1986.

WASSERMANN, J. C. de F.; CRAPEZ de A. C. Efeitos da poluição por óleo em sedimentos da Área de Proteção Ambiental de Guapimirim, RJ. Pós-Graduação em Ciência Ambiental e Instituto de Biologia, Universidade Federal Fluminense. Disponível em: $<$ http://www.uff.br/remadsuff/Biblioteca\%20Virtual.htm > Acesso em: 09 jun. 2006.

WISNIEWSKI, C.; BITTENCOURT, E.; CURCIO, G. R.; RACHWAL, M. F. G. Caracterização nutricional de espécies nativas plantadas sobre solos aluviais do litoral paranaense. In: CONGRESSO E EXPOSIÇÃO INTERNACIONAL SOBRE FLORESTAS - FOREST 99, 5., 1999, Curitiba, PR. Anais... [S.1.: s.n.], 1999. 1 CD-ROM. 